\title{
Pathology and Clinical Research
}

Open Access

ORIGINAL ARTICLE

\section{A New Grading for Clear Cell Renal Cell Carcinoma: Combined WHO/ISUP Grading System}

\author{
Nazlı Sena Seker ${ }^{1 *}$, Ozan Bozkurt' ${ }^{2}$, Güven Aslan', Elif Gokce Devecioglu ${ }^{1}$ and Emine Burçin Tuna ${ }^{3}$ \\ ${ }^{1}$ Department of Pathology, Gazi Yasargil Research \& Training Hospital, Turkey \\ ${ }^{2}$ Department of Urology, Dokuz Eylül University Medical Hospital, Turkey \\ ${ }^{3}$ Department of Pathology, Dokuz Eylül University Medical Hospital, Turkey
}

*Corresponding author: Nazlı Sena Seker, Department of Pathology, Gazi Yasargil Research \&Training Hospital, Talaytepe, Üçkuyular location Kayapınar/Diyarbakır, 21010, Turkey, Tel: +90-5067-389-581

\begin{abstract}
Background: Several parameters have been proposed to predict prognosis in renal cell carcinomas. Although the tumor grade is an important prognostic data in the RCC, there is a low correlation between the observers. The aim of this study was to establish a new grading system based on the combination of different tumor grades in the tumor and the resulting data show the relationship with parameters that are prognostic significance.

Methods: 185 cases of clear cell RCC treated between 2000 and 2013 were included in this study. Demographic features of the disease were obtained from prognostic data from patient records and pathology reports. In fact, the current WHO/ISUP grading was performed by a uropathologist (BT) and a surgical pathology resident (SŞ), and the presence of necrosis in the tumor was also assessed. Combined WHO/ISUP grading system has been applied for tumors with one or more degrees. Combined grade scores are grouped as low, intermediate and high. The relationship between new grades and prognostic parameters was investigated statistically. Pearson Chi-square test was used for the analyzes. It was also assessed by the kappa statistical test between the two observers and within the observers.
\end{abstract}

Results: According to the Combined WHO/ISUP grading system, $39(21 \%)$ of the 185 cases had the same degree and the remaining 146 (79\%) cases had more than one degree. According to the combined grade score group, 77 low grade, 85 intermediate and 29 high grade were evaluated. The agreement between the two observers is "good". Uropathologist was found to be "perfect" in itself, and "good" in surgical pathology resident.

Conclusion: An ideal grading system should have objective criteria, be applicable and repeatable. We think that we have solved the observer inconsistency in large measure by including different grades in the tumor in our study and by including the threshold value we have included in the grading system.

\section{Keywords}

Renal cell carcinoma, Tumor heterogeneity, WHO/ISUP grading system

\section{Abbreviations \\ RCC: Renal Cell Carcinoma}

\section{Introduction}

Newly diagnosed cancer cases throughout the world, $2 \%$ of renal origin [1]. Among the tumors of the urogenital system, $85 \%$ of kidney cancers, which rank third after the prostate and bladder, are renal cell carcinomas (RCC), $12 \%$ of them are urothelial carcinomas originating from the pelvicalyceal system, and the rest are rare malignancies [2,3]. According to WHO 2016, RCCs are divided into 14 subtypes. These; clear cell RCC, multilocular cystic renal cell neoplasm of low malignant potential, papillary RCC, chromophobe RCC, collecting duct carcinoma, renal medullary carcinoma, Mit family translocation RCC succinate dehydrogenase-deficient RCC, mucinous tubular and spindle cell carcinoma, tubulocystic RCC, acquired cystic disease-associated RCC, clear cell papillary RCC and unclassified RCC [4].

The Tumor Lymph Node Metastasis (TNM) staging system of the American Cancer Committee (AJCC) is

Citation: Seker NS, Bozkurt O, Aslan G, Devecioglu EG, Tuna EB (2020) A New Grading for Clear Cell Renal Cell Carcinoma: Combined WHO/ISUP Grading System. Int J Pediatr Res 6:116. doi.org/10.23937/2469$5807 / 1510116$

Accepted: October 20, 2020: Published: October 22, 2020

Copyright: (C) 2020 Seker NS, et al. This is an open-access article distributed under the terms of the Creative Commons Attribution License, which permits unrestricted use, distribution, and reproduction in any medium, provided the original author and source are credited. 
recommended for renal cell carcinoma. This system, which has been modified many times over the past years, was last updated in 2017 [5] and has been shown in many studies that the TNM system is an excellent prognostic factor for patients with RCC [6]. According to the TNM staging system, tumor size, perinephric adipose tissue invasion, renal sinus invasion, major vein invasion (including renal vein and its branches and vena cava), and Gerota fascia invasion (including ipsilateral adrenal invasion) are used to determine the tumor stage [5].

The assessment of the grade of renal cell carcinoma is more problematic. Many grading systems have been proposed for RCC since the 1970s. The study of Skinner, et al. is the first classification that classifies RCC according to its nuclear features, and they reported the highest grade in the tumor as the tumor grade [7]. In 1982, Fuhrman, et al. proposed a 4-tiered grading system determined by the first 3 degrees, nucleus size, nucleus shape, and nucleolar prominence [8]. Among the proposed grading systems, Fuhrman grading system was the most widely used. However, as our knowledge about RCC subtypes increased and larger studies were conducted, the limitations of the Fuhrman grading system were better understood and the prognostic significance began to be questioned. Finally, the World Health Organization (WHO)/International Society of Urological Pathology (ISUP) grading system based on nuclear and nucleolar features have been developed [9]. In fact, computerized automated grading systems have even been applied to increase the compatibility between researchers $[9,10]$.

An ideal grading system should be objective, practi- cal and reproducible. The aim of this study is to create a new grading system for CCRCC, considering different tumor grades within the tumor, and to investigate its practicality and reproducibility and its effect on prognostic factors.

\section{Methods}

This study included 185 clear cell RCC cases diagnosed at Dokuz Eylul University Medical Faculty Hospital between 2000 and 2013. Papillary and chromophobe renal cell carcinomas and other kidney tumor subtypes were not included in this study. Demographic features, tumor Fuhrman grade, tumor size, presence of microvascular invasion (MVI), pathological tumor stage, type of operation, presence of distant metastases were obtained from patient records and pathology reports. Hematoxylin \& Eosin (H\&E) stained preparations of $4 \mu \mathrm{m}$ thickness were graded according to the current WHO/ ISUP by a uropathologist (BT) and a surgical pathology resident (SS) regardless of the initial tumor Fuhrman grade [11]. According to this grading system; the cases where the nucleoli are basophilic and absent or inconspicious at $\mathrm{x} 400$ magnification are grade 1 (Figure 1A), the cases where the nucleoli are seen as eosinophilic at x400 magnification but not prominent at $x 100$ magnification are grade 2 (Figure $1 \mathrm{~B}$ ) and the cases where the nucleoli conspicuous and eosinophilic at $\times 100$ magnification are clearly visible tumors were graded as grade 3 (Figure 1C). Tumors with sarcomatoid (Figure 1D) or rhabdoid (Figure 1E) differentiation or tumor giant cells (Figure 1F) or with pronounced nuclear pleomorphism were accepted as grade 4 . Before the evalution, the cases representing WHO/ISUP grades 1, 2, 3 and 4 were
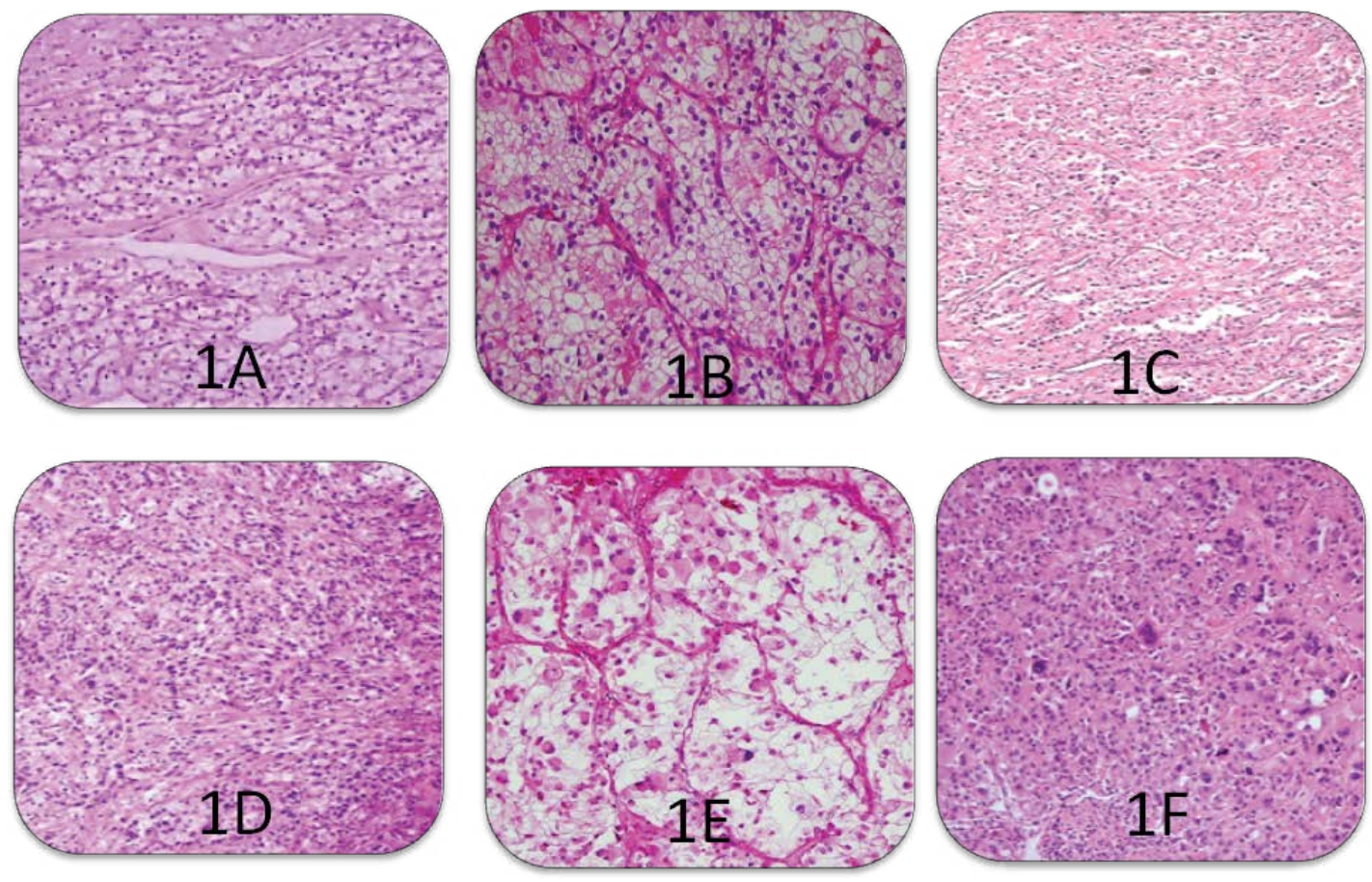

Figure 1: (A)WHO/ISUP grade 1 (H\&E, x200) in clear cell RCC; (B) WHO/ISUP grade 2 (H\&E, x200) in clear cell RCC; (C) WHO/ISUP grade 3 (H\&E, x100) in clear cell RCC; (D) WHO/ISUP Grade 4, sarcomatiod differentiation (H\&E, 200); (E) WHO/ISUP Grade 4, rabdoid differentiation (H\&E, 200); (F) WHO/ISUP Grade 4, tumour giant cells (H\&E, 200). 

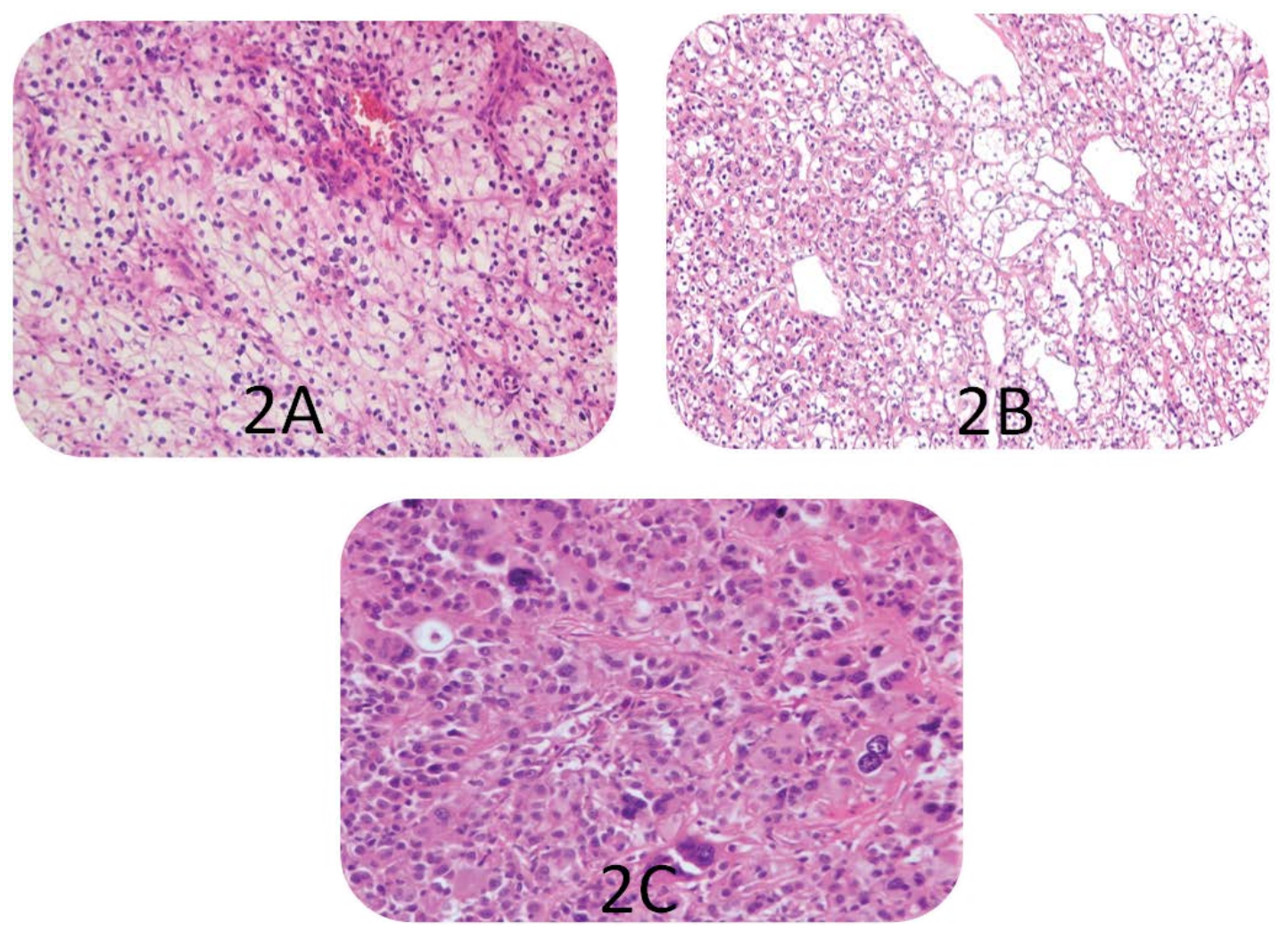

Figure 2: (A) Combined grade score $2+1$ in clear cell RCCs (H\&E, x200); (B) Combined grade score $3+2$ in clear cell RCCs (H\&E, x200); (C) Combined grade score $3+4$ in clear cell RCCs (H\&E, x200).

evaluated together by the observers according to the criteria defined in the literature. This assesment was made considering tumor heterogeneity. The combined grading system for tumors containing one or more grades is based on the criteria of the Gleason grading system [12]. According to this grading system, the most common grade in the tumor was considered primary and the second most common grade was considered secondary. If the second most common tumor grade rate was $5 \%$, it was considered secondary. In addition, in tumors with more than 2 degrees, the most common grade is considered primary, the highest grade tumor area is the secondary degree if it fills a $\times 400$ magnification area [13]. In this case, the tumor with a low grade was ignored and was not included in the grading. The agreement within the observer and between the observers was evaluated with 25 randomly selected cases. The second evaluation was made at least 4 weeks after the first evaluation. The combined WHO/ISUP grades are grouped as low, intermediate and high according to the combined grade score. Tumors with a combined WHO/ISUP grade score of $2-4(1+1 / 1+2 / 2+1 / 2+2)$ (Figure 2A) are low, and tumours with $5(2+3 / 3+2)$ (Figure 2B) are intermediate and tumors with $6,7,8(3+$ $3 / 2+4 / 4+2 / 3+4 / 4+3 / 4+4$ ) (Figure $2 C$ ) are grouped as high grade. In addition, the presence of necrosis in the tumor was examined in order to determine its relationship with the tumor grade. Statistical analysis of the data was done with SPSS 22.0 program. Percentage values for the descriptive characteristics of all patients were taken. Pearson chi-square test was used to determine whether there is a difference between the combined WHO/ISUP grades in terms of gender, tumor size, pathological tumor stage, necrosis, MVI, metastasis and death. According to the evaluation results, if $p$ value is equal to or less than 0.05 , it is considered statistically significant. The correlation between the combined WHO/ISUP grading system and the WHO/ISUP grading system was evaluated using Kendall's tau-b test.

The agreement within the observer and between the observers was evaluated with the kappa statistical test, and the kappa values were accepted as: Mild agreement $<0.40$; good agreement $=0.40-0.75$; perfect agreement $>0.75[14,15]$.

\section{Results}

Of the 185 cases we included in the study, 117 (63.2\%) were male and $68(36.8 \%)$ were female, and the average patient age was 59.6 ( $\mathrm{min} 27-\mathrm{max} 87$ ). According to the tumor staging system; 120 cases in T1 stage, 25 cases in T2 stage, 29 cases in T3 stage and 1 case in T4 stage were determined.

According to Kendall's tau-b correlation test, $r$ value between combined WHO/ISUP and WHO/ISUP grading system was determined as 0.76 . It shows that there is a strong correlation between the two grading systems.

According to the combined WHO/ISUP grading system, a total of 4 disparities were established among observers. In the first evaluation, the agreement between 
Table 1: Combined WHO/ISUP grading and combined grading score groups.

\begin{tabular}{|l|l|l|l|l|}
\hline Primary grade & Secondary grade & Combined grade score & No & $\begin{array}{l}\text { Combined grading score } \\
\text { groups }\end{array}$ \\
\hline 1 & 1 & 2 & 16 & Low \\
\hline 1 & 2 & 3 & 13 & Low \\
\hline 2 & 1 & 3 & 27 & Low \\
\hline 2 & 2 & 4 & 15 & Low \\
\hline 2 & 3 & 5 & 40 & Intermediate \\
\hline 3 & 2 & 5 & 45 & Intermediate \\
\hline 3 & 3 & 6 & 8 & High \\
\hline 4 & 2 & 6 & 3 & High \\
\hline 2 & 4 & 6 & 2 & High \\
\hline 3 & 4 & 7 & 12 & High \\
\hline 4 & 3 & 7 & 4 & High \\
\hline
\end{tabular}

Table 2: Combined grade score groups, death and metastasis relationship.

\begin{tabular}{|l|l|l|l|l|l|l|}
\hline \multicolumn{2}{c}{} & \multicolumn{2}{c}{ Metastasis } & \multicolumn{3}{l|}{ Death } \\
\cline { 3 - 7 } & & No & Yes & No & Yes \\
\hline $\begin{array}{l}\text { Combined grade } \\
\text { score groups }\end{array}$ & Low (1-4) & Num & 35 & 1 & 33 & 3 \\
\cline { 2 - 7 } & Intermediate (5) & Num & 70 & 16 & 63 & 24 \\
\cline { 2 - 7 } & High (6-8) & Num & 41 & 21 & 35 & 27 \\
\hline Total & Num & 146 & 38 & 131 & 54 \\
\hline
\end{tabular}

Chi square test respectively; $p=0.001, p=0.001$

the two observers was found to be "good" ( $\mathrm{k}=0.603)$. In the second evaluation, a difference was observed in 3 cases. The agreement between the observers was found to be "good" ( $\mathrm{k}=0.667$ ). It was noted that there was no disparity in high-grade tumors among observers.

When we look at the agreement of the observer in itself, in the evaluation of the uropathologist, there was a difference in 3 cases that did not change the combined grade score group. When we look at the agreement of the surgical pathology resident, changes were detected in a total of 4 cases. The agreement of the uropathologist within itself was found to be "perfect" $(k=1.00)$, and the agreement of the surgical pathology resident was found to be "good" ( $\mathrm{k}=0.630)$. According to the combined WHO/ISUP grading system, the evaluation results of 185 cases are presented in Table 1 . In the combined WHO/ISUP grading system, 20 (29.4\%) of the female patients were low-grade, 30 were intermediate-grade (44.1\%), 18 (26.5\%) were high-grade; in male patients, $16(13.7 \%)$ are low, 58 are intermediate (48.7\%), 44 (37.6\%) are high. Male patients have a higher grade tumor at the time of diagnosis $(p=0.027)$. A statistically significant relationship was found between the combined WHO/ISUP grading system and death, metastasis (Table 2). According to the combined grade score groups, MVI was found in 2 of 34 low grade cases, MVI in 14 of 73 intermediate grade cases and 34 of 44 high-grade cases. Accordingly, as the combined grade score increases, mortality, metastasis and MVI rates increase $(p=0.001, p=0.001$ and $p=0.011$, respectively).
The tumor diameter of the cases varies between $1.2 \mathrm{~cm}$ and $18 \mathrm{~cm}$, and the average tumor size is 4.5 $\mathrm{cm}$ in low-grade tumors, 5.2 in intermediate-grade tumors and 7.1 in high-grade tumors. There is a statistically significant difference between tumor grade and tumor size $(p<0.001)$. Similarly, there is a statistically significant relationship between necrosis and tumor grade ( $p<0.001) .30(71 \%)$ of 42 cases with necrosis were high grade and $32(22.4 \%)$ of 143 cases without necrosis were high grade. Of the patients with pathological tumor stage 1, 28 were low, 62 were intermediate and 30 were high combined WHO/ISUP grade. Of the cases with pathological tumor stage 3, 2 were low, 12 were intermediate and 25 were high combined WHO/ ISUP grade. As a result, tumors with a high WHO/ISUP grade were found to be in the advanced tumor stage $(p<0.001)$. Of the WHO/ISUP grade 1 tumors $(n=22)$, 18 were low-grade; 4 of them are intermediate-grade according to the combined WHO/ISUP grading system. According to the combined WHO/ISUP grading system; of the WHO/ISUP grade 2 tumors $(n=77), 13$ were low, 56 were intermediate and 8 were high grade; 5 of WHO/ ISUP grade 3 tumors $(n=67)$ are low, 21 are intermediate, 41 are high grade and of the WHO / ISUP grade 4 tumors $(n=19), 6$ were intermediate and 13 were high grade.

\section{Discussion}

Renal cell carcinomas are the most common malignancy of the kidney in humans [16]. The most common 
histological type among renal cell carcinomas is clear cell RCC and it is approximately $85 \%$ of all renal cell carcinomas [17].

The tumor stage is the most important prognostic parameter for RCCs has been proven in many studies $[18,19]$ and the histological grade of the tumor is the most important prognostic factor after the stage. Many studies show that the Fuhrman grade is an independent prognostic factor for survival $[13,20]$. Although the nuclear size measurement in Fuhrman system, which is a system based on the nuclear features of tumor cells, seems to support the objectivity of the grading, pathologists have been shown to predict the nuclear size by nucleoli prominence [21,22]. In addition, it is stated that the 3 parameters (nuclear size, nucleolar prominence and nuclear irregularity) determined during Fuhrman grading are not always parallel and grading is not healthy when there are different features within the same tumor $[23,24]$. Many studies have researched 2 and 3 tiered modified Fuhrman grading systems instead of the conventional 4 tiered grading system $[25,26]$. In a study of 431 BHBHK cases, 3 systems including conventional Fuhrman grading system were compared [25]. In one of these grading systems, the 2 tiered grading system is that Fuhrman grade 1 and 2 in the same group, 3 and 4 in the same group $(1+2 / 3+4)$ and the 3 tiered grading system is grades 1 and 2 are separate, 3 and 4 in the same group has been made $(1 / 2 / 3+4)$. The three grading system about Fuhrman was compared in terms of survival. Of these, only the 3 tiered grading system $(1 / 2 / 3+4)$ was found statistically significant in multiple analyzes in terms of predicting disease-free survival [25]. Qayyum, et al. studied the differences in predicting prognosis by modifying the Fuhrman grading system in their study with 237 clear cell RCC cases. They compared 5 different modified Fuhrman grading systems. In these grading systems, their grades are grouped as $1+2 / 3+4$, $1 / 2+3+4,1+2+3 / 4,1 / 2 / 3+4$ and $1+2 / 3 / 4$. Among these systems, the system in which only the grades 1 and 2 grouped 3 and 4 separately $(1+2 / 3 / 4)$ was found meaningful for cancer-specific survival. In addition, the system where grade 1,2 and 3 were grouped as the same and grade 4 as separate $(1+2+3 / 4)$ was found to be significant in terms of predicting the pathological T stage [26]. In Fuhrman's original study, the majority of cases, grade 2 and 3 tumors, were grouped and compared with grade 1 tumors. As a result, grade 1 tumors have been shown to have a much better prognosis [8].

In our study, tumors with a combined grade score of $2-4(1+1 / 1+2 / 2+1 / 2+2)$ were grouped as low grade, and tumors with a combined grade score of $5(2+3 / 3$ +2 ) were moderately. Since WHO/ISUP grade 4 tumors were significantly associated with poor prognosis, they also evaluated tumors with a combined grade score of 2 +4 or $4+2$ as high grade. Tumors with a combined grade score of $3+3$ were included in the high grade group due to the high rate of metastasis, $\mathrm{MVI}$, necrosis and death. Significant differences were observed between the combined grade score groups in terms of metastasis, death, MVI, tumor stage and necrosis. Although the combined grade score group did not show a significant difference in tumor size in low and intermediate grade tumors, it was noteworthy that high-grade tumors were significantly larger tumors.

One of the important reasons for the search for alternative grading system related to prognosis for RCCs is the difficulty of application of Fuhrman grading and low agreement among pathologists [27-30]. In the study of Ficarra, et al. with 388 RCC cases, a pathologist specializing in uropathology re-evaluated the cases, regardless of the degree written in the original report. In this study, the intra-observer agreement was evaluated, the agreement between the two grades was found to be "medium" (k value 0.44). In this study, it was shown that mismatch was mostly caused by grade 1 and 2 tumors [31]. In a study involving 3 researchers, in which the compatibility between observers was investigated in the Fuhrman grading system, the agreement between the researchers in the conventional 4 tiered system was found as "low-medium" ( $k=0.22)$. It was found that the agreement between the 3 pathologists, which they did by grouping grades 1 and 2 in the same group and grades 3 and 4 separately was slightly better ( $\kappa=$ 0.34 ). However, the most satisfactory result is that the groups 1 and 2 are the same and the 3 and 4 the same $(1+2 / 3+4)$ grouped in the 2 tiered grading system they made $(k=0.44)$ [28]. Similarly, in another study evaluating the compatibility between 4 pathologists, the inter observer and intraobserver agreement according to the conventional Fuhrman grading system was found to be "medium" ( $\mathrm{k}=0.29$ and $\mathrm{k}=0.45$ respectively). In the modified Fuhrman grading system, which they group grades 1 and 2 as low, and grades 3 and 4 as high, the inter-observer agreement is determined as "medium" $(\mathrm{k}=0.45)$, while the intra-observer agreement is "good" ( $k=0.53)$ [22].

Reasons of low agreement between observers are shown to be primary tumor heterogeneity, inappropriate tissue fixation and lack of a standard threshold that should be when increasing tumor grade [30]. Although the WHO/ISUP grading system used today is based on more objective data than the Fuhrman grading system, we think that the inter-observer agreement problem may still continue due to the fact that clear cell RCCs are heterogeneous tumors. The most important reason for this may be that tumors contain more than one grade, which has been shown in many studies. The study of Al-Aynati, et al. [20] and the study of Bektaş, et al. [25] also support this situation. Similarly, in our study, 39 cases $(21.1 \%)$ were the same, 131 cases $(70.8 \%)$ were found to have 2 different grades and 15 cases $(8.1 \%)$ included 3 different grades According to the combined WHO/ISUP grading system, which was performed con- 
sidering the tumor heterogeneity, the agreement between the observers was found as "good" $(\kappa=0.641)$. When we look at the intra-observer agreement, the agreement of the uropathologist was found to be "perfect" and the agreement of surgical pathology resident was "good" ( $k=1.00$ and 0.630 , respectively).

In another study, cell type, tumor grade and Ki67 proliferative index were found to be heterogeneous in clear cell RCC with tumor size greater than $3.8 \mathrm{~cm}$ [32]. The histological and molecular heterogeneity of the tumor also leads to differences in the clinical responses of the cases [33]. For this reason, many new molecular studies are carried out for targeted therapies and prognostic parameters in recent studies [17,34].

In our study, we aimed to resolve the observer disparity by including different grades contained in the tumor into the grading system and standardizing the threshold value. As the need for a new grading system that reflects the morphological heterogeneity of the tumor and has high reproducibility, increases day by day, studies for an ideal grading system are continuing. In the future, combining with molecular markers and doing new studies could solve this problem completely.

\section{Acknowlegdments}

The authors declare no conflicts of interest.

\section{Authors Contribution}

All authors contributed to the design and implementation of the research, to the analysis of the results and to the writing of the manuscript.

\section{References}

1. Parkin DM, Whelan SL, Ferlay J, Teppo L, Thomas DB (2002) Cancer incidence in five continents. IARC Scient Publ 8.

2. Ljungberg B, Steven C Campbell, Han Yong Choi, Didier Jacqmin, Jung Eun Lee, et al. (2011) The epidemiology of renal cell carcinoma. Eur Urol 60: 615-621.

3. Howlader N (2011) Surveillance, epidemiology, and end results program. Bethesda: National Cancer Institute.

4. Moch $\mathrm{H}$, Antonio L Cubilla, Peter A Humphrey, Victor E Reuter, Thomas M Ulbright (2016) WHO Classification of Tumours of the Urinary System and Male Genital Organs. 8.

5. Robins DJ, Alexander C Small, Mahul B Amin, Bernard H Bochner, Sam S Chang, et al. (2017) MP86-17 THE 2017 american joint committee on cancer eighth edition cancer staging manual: Changes in staging guidelines for cancers of the kidney, renal pelvis and ureter, bladder, and urethra. The Journal of Urology 197: e1163.

6. Gettman MT, ML Blute (2002) Update on pathologic staging of renal cell carcinoma. Urology 60: 209-217.

7. Skinner DG, RB Colvin, CD Vermillion, RC Pfister, WF Leadbetter (1971) Diagnosis and management of renal cell carcinoma A clinical and pathologic study of 309 cases. Cancer 28: 1165-1177.

8. Fuhrman SA, LC Lasky, C Limas (1982) Prognostic significance of morphologic parameters in renal cell carcinoma. Am J Surg Pathol 6: 655-663.
9. Katherine Tian, Christopher A Rubadue, Douglas I Lin, Mitko Veta, Michael E Pyle, et al. (2019) Automated clear cell renal carcinoma grade classification with prognostic significance. PLoS One 14: e0222641.

10. Yeh FC, Anil V Parwani, Liron Pantanowitz, Chien Ho (2014) Automated grading of renal cell carcinoma using whole slide imaging. J Pathol Inform 5: 23.

11. Dagher J, Brett Delahunt, Nathalie Rioux-Leclercq, Lars Egevad, John R Srigley, et al. (2017) Clear cell renal cell carcinoma: Validation of World Health Organization/International Society of Urological Pathology grading. Histopathology 71: 918-925.

12. Epstein JI, Lars Egevad, Mahul B Amin, Brett Delahunt, John R Srigley, et al. (2016) The 2014 International Society of Urological Pathology (ISUP) consensus conference on Gleason grading of prostatic carcinoma. Definition of grading patterns and proposal for a new grading system. Am J Surg Pathol 40: 244-252.

13. Frank I, Michael L Blute, John C Cheville, Christine M Lohse, Amy L Weaver, et al. (2002) An outcome prediction model for patients with clear cell renal cell carcinoma treated with radical nephrectomy based on tumor stage, size, grade and necrosis: The SSIGN score. J Urol 168: 23952400.

14. Fleiss JL, B Levin, MC Paik (2013) Statistical methods for rates and proportions. John Wiley \& Sons.

15. Rosner B (2015) Fundamentals of biostatistics. Nelson Education.

16. Chow WH, LM Dong, SS Devesa (2010) Epidemiology and risk factors for kidney cancer. Nat Rev Urol 7: 245-257.

17. Huang J, Zhou Li, Lijun Fu, Dahe Lin, Chunhua Wang, et al. (2020) Comprehensive characterization of tumor mutation burden in clear cell renal cell carcinoma based on the three independent cohorts. J Cancer Res Clin Oncol.

18. Tsui KH, O Shvarts, RB Smith, RA Figlin, JB de Kernion, et al. (2000) Prognostic indicators for renal cell carcinoma: a multivariate analysis of 643 patients using the revised 1997 TNM staging criteria. J Urol 163: 1090-1095.

19. Klatte T, SH Rossi, GD Stewart (2018) Prognostic factors and prognostic models for renal cell carcinoma: A literature review. World J Urol 36: 1943-1952.

20. BC Andreiana, AE Stepan, C Mărgăritescu, AM AI Khatib, MM Florescu, et al. (2018) Histopathological prognostic factors in clear cell renal cell carcinoma. Curr Health Sci J 44: 201-205.

21. Lanigan D, R Conroy, C Barry Walsh, B Loftus, D Royston, et al. (1994) A comparative analysis of grading systems in renal adenocarcinoma. Histopathology 24: 473-476.

22. Al-Aynati M, Vicky Chen, Samih Salama, Hafez Shuhaibar, Darin Treleaven, et al. (2003) Interobserver and intraobserver variability using the Fuhrman grading system for renal cell carcinoma. Arch Pathol Lab Med 127: 593-596.

23. Delahunt B (2009) Advances and controversies in grading and staging of renal cell carcinoma. Mod Pathol 22: S24-S36.

24. Delahunt B, Lars Egevad, Hemamali Samaratunga, Guido Martignoni, John N Nacey, et al. (2016) Gleason and Fuhrman no longer make the grade. Histopathology 68: 475-481.

25. Hong SK, Chang W Jeong, Ji H Park, Hyung S Kim, Cheol Kwak, et al. (2011) Application of simplified Fuhrman grading system in clear-cell renal cell carcinoma. BJU Int 107: 409-415. 
26. Qayyum T, Tahir Qayyum, Peter McArdle, Clare Orange, Morag Seywright, Paul Horgan, et al. (2013) Reclassification of the Fuhrman grading system in renal cell carcinoma-does it make a difference? Springerplus 2: 378.

27. Bektas S, Burak Bahadir, Nilufer Onak Kandemir, Figen Barut, Aylin Ege Gul, et al. (2009) Intraobserver and interobserver variability of fuhrman and modified fuhrman grading systems for conventional renal cell carcinoma. The Kaohsiung Journal of Medical Sciences 25: 596-600.

28. Lang $H$, Véronique Lindner, Marc de Fromont, Vincent Molinié, Hervé Letourneux, et al. (2005) Multicenter determination of optimal interobserver agreement using the Fuhrman grading system for renal cell carcinoma: Assessment of 241 patients with > 15-year follow-up. Cancer 103: 625-629.

29. Medeiros LJ, AB Gelb, LM Weiss (1998) Renal cell carcinoma. Prognostic significance of morphologic parameters in 121 cases. Cancer 61: 1639-1651.
30. Novara G, Guido Martignoni, Walter Artibani, Vincenzo Ficarra (2007) Grading systems in renal cell carcinoma. The Journal of Urology 177: 430-436.

31. Ficarra V, Guido Martignoni, Nicola Maffei, Matteo Brunelli, Giacomo Novara, et al. (2005) Original and reviewed nuclear grading according to the Fuhrman system: A multivariate analysis of 388 patients with conventional renal cell carcinoma. Cancer 103: 68-75.

32. Zaldumbide L, Asier Erramuzpe, Rosa Guarch, Jesús M Cortés, José I López (2015) Large (> $3.8 \mathrm{~cm}$ ) clear cell renal cell carcinomas are morphologically and immunohistochemically heterogeneous. Virchows Arch 466: 61-66.

33. Rabjerg M (2017) Identification and validation of novel prognostic markers in renal cell carcinoma. Dan Med J 64: B5339.

34. Ishimaru T (2020) Molecular components of the RCC Grade. Seminars in Nephrology 40: 14-27. 